Case Report

\title{
Development of Localized Pulmonary Interstitial Emphysema in a Late Preterm Infant without Mechanical Ventilation
}

\author{
Pritish Bawa, ${ }^{1}$ Kultida Soontarapornchai, ${ }^{2}$ Agnes Perenyi, \\ Rachelle Goldfisher, ${ }^{1}$ and John Amodio ${ }^{1}$ \\ ${ }^{1}$ Department of Radiology, SUNY Downstate Medical Center, 450 Clarkson Avenue, Brooklyn, NY 11203, USA \\ ${ }^{2}$ Department of Pediatrics, SUNY Downstate Medical Center, 450 Clarkson Avenue, Brooklyn, NY 11203, USA
}

Correspondence should be addressed to John Amodio; john.amodio@downstate.edu

Received 9 December 2013; Accepted 23 January 2014; Published 11 March 2014

Academic Editors: D. Fischer and G. Montini

Copyright (c) 2014 Pritish Bawa et al. This is an open access article distributed under the Creative Commons Attribution License, which permits unrestricted use, distribution, and reproduction in any medium, provided the original work is properly cited.

Pulmonary interstitial emphysema (PIE) is not an uncommon finding in premature infants with respiratory distress who need respiratory support by mechanical ventilation. PIE has been reported in a few cases of neonates in whom either no treatment other than room air was given or they were given continuous positive end-expiratory pressure (CPAP) support. We present a case of a premature neonate who presented with respiratory distress, in whom PIE and spontaneous pneumothorax (PTX) developed while on CPAP therapy only. The patient was treated conservatively with subsequent resolution of the radiological findings and clinical improvement. No surgical intervention was required. It is important to know that PIE may develop independently of mechanical ventilation. We would like to add this case to the literature and describe the pertinent plain film and computed tomography (CT) findings of this entity, the possible mechanism of development, and the differential diagnosis. A review of the literature is also provided.

\section{Introduction}

Pulmonary interstitial emphysema (PIE) is a form of air leak syndrome. It occurs when the air escapes from the small airway or alveolus into the pulmonary interstitium. It dissects along the bronchovascular bundles and radiates outwards to the periphery of the lung to form subpleural blebs. These blebs may burst through visceral pleura into the pleural space resulting in pneumothorax (PTX). The interstitial air can also pass through the lung hilus directly into mediastinum and cause pneumomediastinum. Further grave complications of interstitial air can be pneumopericardium, pneumoperitoneum, subcutaneous emphysema, and terminally massive air embolism [1].

When infants are given positive pressure ventilation, especially but not exclusively by intubation and mechanical ventilation, a continuous pressure ensures passage of air throughout the interstitial lymphatics. The incidence of PIE is high in ventilated low-birth-weight infants, reported as high as 33\% in this group by Greenough and others [2-4]. PIE is more common in premature infants with surfactant deficiency treated with respiratory support in the form of mechanical ventilation.

The incidence of spontaneous air leak syndromes in premature infants is about $1-2 \%$; many infants are asymptomatic. There are few cases of spontaneous PIE in patients on continuous positive airway pressure (CPAP) [4-7].

We wish to add an additional report of a late preterm infant who developed localized pulmonary interstitial emphysema within the right lung, in association with PTX and pneumomediastinum, while on CPAP therapy. We also review the literature on this topic.

\section{Case Report}

A 36-week and 5-day gestation male infant was born to a mother 30 years of age via Cesarean section $(C / S)$ due to repeated $\mathrm{C} / \mathrm{S}$. The mother's pregnancy was complicated by preeclampsia. All of her serology was negative, while inadequate treatment for positive GBS, without premature rupture of membranes, was reported. The infant's Apgar 
scores were 8 and 8 at 1 and 5 minutes, respectively. After 5 minutes of life, he developed nasal flaring, grunting, and subcostal retractions. He was placed on nasal CPAP, with oxygen saturation of $30 \%$ and PEEP of 5.

Initial chest X-ray (CXR) (Figure 1) demonstrated diffuse airspace disease and mild hyperinflation. The infant subsequently developed tachypnea and dyspnea, with subcostal, intercostal, and substernal retractions, and required higher oxygen saturation of $40 \%-45 \%$ to maintain oxygen saturation above 95\%. A sepsis workup was negative. CXR, at that time, showed the development of multiple air- containing structures within the right lung and pneumomediastinum (Figure 2). A small pneumothorax was seen on a left side down decubitus view. The patient remained stable on CPAP, but subsequent $\mathrm{X}$-rays of the chest demonstrated the development of multiple air-containing cavity-like lesions involving only the right lung and a persistent small pneumothorax (Figure 3). As the patient had not received mechanical ventilation, the diagnosis of a congenital pulmonary airway malformation (CPAM) was considered. A CT scan of the chest at that time revealed multiple linear collections of air following a perivascular distribution, diagnostic of PIE, and loculated pneumomediastinum (Figure 4). The patient remained in stable condition and he was placed in room air; the PIE resolved spontaneously while the patient was in room air. The initial airspace disease was thought to be secondary to retained fetal lung fluid.

\section{Discussion}

PIE and its pathophysiology, both in children and adults, were first described by M. T. Macklin and C. C. Macklin in 1944 [8]. Risk factors for air leak syndromes include prematurity, surfactant deficiency syndrome, meconium aspiration syndrome, amniotic fluid aspiration, infection, low Apgar score, or the need for positive pressure ventilation (PPV) during resuscitation at birth and use of high peak airway pressures on mechanical ventilation.

Preterm infants are at an increased risk for PIE because the perivascular connective tissue is more abundant and less dissectible in preterm than older infants. This allows air trapping in the perivascular space $[9,10]$.

Lungs with PIE are stiff and the interstitial air can compress adjacent functional lung tissue and vascular structures hampering proper ventilation and pulmonary blood flow. Thus, early detection of this entity is very important to prevent further damage by ventilation under pressure. This sequence of events may be rapid in infants more severely afflicted with surfactant deficiency as well as in those who need high ventilatory pressure for adequate oxygenation.

Findings from animal studies have suggested that PIE may be more associated with structural and maturational factors rather than with overdistention alone [11]. This can explain the occurrence of this entity in premature infants without history of mechanical ventilation.

Very low birth weight ( $\leq 1500$ grams) is an independent risk factor [12]. In those premature infants, PIE can occur at low mean airway pressure and probably reflects the underdeveloped lung's increased sensitivity to stretch. In a retrospective case-controlled study, 11 (24\%) of 45 extremely lowbirth-weight ( $\leq 1,000$ grams) infants developed pulmonary interstitial emphysema [13]. All infants included in the study were treated with a conventional ventilator before the onset of pulmonary interstitial emphysema.

PIE has been described as local or diffuse and acute or persistent [14]. Persistent cases of PIE are categorized pathologically by the presence of giant cells that are seen after ten days of disease $[5,14]$. The localized form generally has larger cysts in one or two lobes, whereas the diffuse form commonly has smaller cysts in all the lobes. Cysts in localized PIE may enlarge and result in compression and atelectasis of the adjacent lung.

A number of adverse side effects and complications of CPAP have been described [10]. It has been noted that air leaks, such as pneumothorax and pneumomediastinum, do occur on CPAP [10]. The mechanism may be related to overdistention of the more compliant areas of the lung, with subsequent dissection of air into the mediastinum or pleura.

There have been only a few cases reported for PIE developing in unventilated neonates, like the one we are reporting. PIE in these cases occurred spontaneously and more commonly with CPAP therapy $[4,5]$.

Freysdottir et al. [15] documented a case of spontaneous PIE in a premature infant born by normal vaginal delivery who was never ventilated. The patient had a complicated course in hospital and subsequently underwent surgery for PIE. The authors proposed another mechanism for the spontaneous development of PIE, namely, laryngomalacia, leading to airway obstruction and subsequent air trapping.

Gurakan et al. [7] described a case of localized persistent PIE in an unventilated very-low-birth-weight premature infant who was on nasal CPAP for 5 days. The infant underwent a CT scan of the chest, which demonstrated multiple thick walled cystic appearing lesions in one lung; since the process was localized to one lung and the patient never received ventilatory support, the diagnosis of a congenital pulmonary airway malformation (CPAM) was considered. However, the process regressed with conservative management.

Bas et al. [9] described a male infant born via spontaneous vaginal delivery at 33 weeks of gestation with a birth weight of 2440 grams. The baby was given CPAP for mild respiratory distress and later developed localized persistent PIE, with slow recovery on conservative management.

Al-Abdi and Singhal [4] described PIE in a premature very-low-birth-weight infant delivered by emergency Cesarean section, who was placed on CPAP. The authors proposed that pregnancy induced hypertension (PIH), hemolysis, elevated liver enzymes, and low platelet count syndrome (HELLP) may be risk factors for PIE. It is interesting that in the case we report there was a history of maternal pregnancy induced hypertension.

Interestingly, a case of spontaneous development of pulmonary interstitial emphysema with PTX and pneumomediastinum complicating pneumonia in a 6-week-old infant was reported by Lee and Im [16]. The patient had no history of 


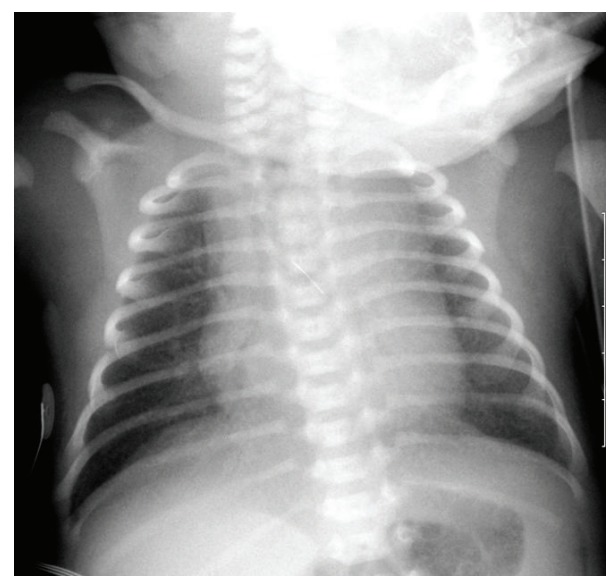

FIGURE 1: Frontal view of the chest demonstrates mild hyperinflation and mild diffuse air-space disease.

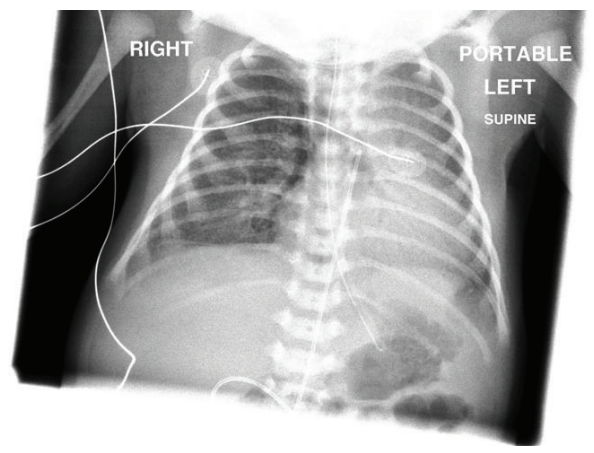

Figure 2: Frontal view of the chest demonstrates the development of multiple air-containing structures within the right lung and pneumomediastinum.

resuscitation, nasal CPAP support, or mechanical ventilation. However, this case was associated with lung infection, which may have weakened the structural support of the lung tissue, allowing PIE to develop more readily. There are a few other case reports of PIE in unventilated infants caused by infection/presumed infection. Four of these cases are reported by Crosswell and Stewart [3], Prusnani et al. [17], O'Donovan et al. [18], and Boisset [19].

We are not certain why only the right lung was involved in our case, but we postulate that whatever pressure effects developed as a result of CPAP may have preferentially affected the right lung due to the more vertical course of the right bronchial anatomy. Gaylord et al. [20] observed PIE occurring bilaterally and of those with unilateral disease, $90 \%$ had PIE on the right.

Management of PIE includes lateral decubitus positioning, selective bronchial intubation or occlusion, intratracheal surfactant administration, and corticosteroids for the benign disease. Tube thoracostomy has also been used. For the more aggressive or persistent form of the disease high frequency oscillation ventilation, extracorporeal membrane oxygenation, pleurotomy, lobectomy, and pneumonectomy may be used. Surgery is usually required for lesions causing

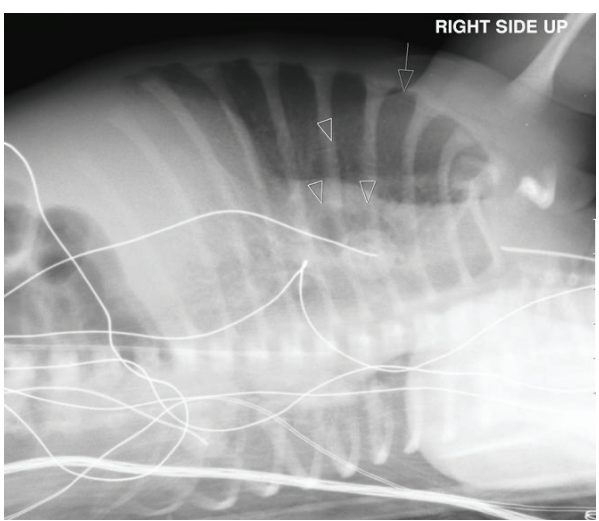

FIgURE 3: Left lateral decubitus view shows small pneumothorax (arrow) and enlargement of air-containing structures within the right lung (arrowhead).

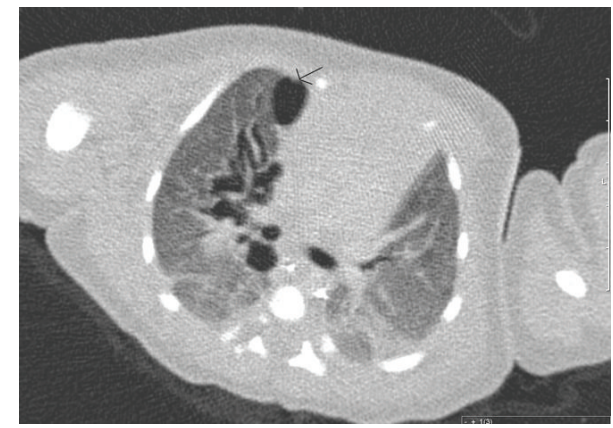

FIGURE 4: Representative slice from CT scan shows loculated pneumomediastinum (arrow) and multiple branching lucencies in a perivascular distribution, compatible with PIE.

pulmonary dysfunction and/or cardiovascular compromise, which do not resolve with more conservative measures. In the case we are reporting, no special maneuvers were necessary, as the PIE rapidly resolved.

The differential diagnosis of an air-containing lesion in a neonate includes congenital and acquired causes, including CPAM, bronchogenic cyst, congenital lobar emphysema, diaphragmatic hernia, lymphangiectasia, cystic lymphangioma, and sequelae to infection. In the case presented the first radiograph did not reveal any air-containing lesions, but there was a rapid development of localized air- containing structures within the right lung. Since the patient was not on mechanical ventilation, the possibility of CPAM was considered. The CT scan was very helpful in distinguishing PIE from other lesions, as it clearly demonstrated air extending along the peribronchovascular bundles.

In summary, we present a case of a full-term infant who was placed on nasal CPAP and developed PIE. This case was also complicated by the development of a small PTX and pneumomediastinum. The PIE, PTX, and pneumomediastinum resolved subsequently while the patient was in room air (Figure 5). One should be aware that PIE may develop spontaneously or with nasal CPAP and is not necessarily a complication of mechanical ventilation. CT scans of the 


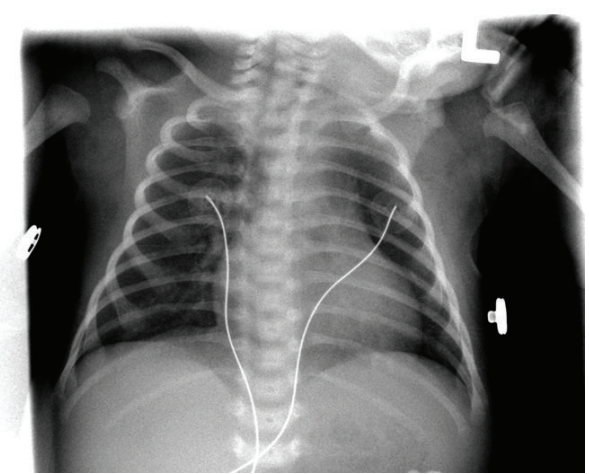

FIGURE 5: Frontal view of the chest shows complete resolution of PIE, pneumothorax, and pneumomediastinum.

chest may help distinguish PIE from other lesions which have a similar appearance on chest X-ray. Conservative management in these cases may lead to complete resolution.

\section{Conflict of Interests}

The authors declare that there is no conflict of interests regarding the publication of this paper.

\section{References}

[1] S. Malkin and B. A. O. Binns, "A case of spontaneous pneumothorax and pneumomediastinum in the newborn," Canadian Medical Association Journal, vol. 86, no. 4, pp. 183-185, 1962.

[2] A. Greenough, A. K. Dixon, and N. R. C. Roberton, "Pulmonary interstitial emphysema," Archives of Disease in Childhood, vol. 59, no. 11, pp. 1046-1051, 1984.

[3] H. E. Crosswell and D. L. Stewart, "Special feature: radiological case of the month. Pulmonary interstitial emphysema in a nonventilated preterm infant," Archives of Pediatrics \& Adolescent Medicine, vol. 155, no. 5, pp. 615-616, 2001.

[4] S. Y. Al-Abdi and N. Singhal, "Pulmonary interstitial emphysema and continuous positive airway pressure in a premature infant," Saudi Medical Journal, vol. 26, no. 10, pp. 1627-1629, 2005.

[5] D. R. Berk and L. J. Varich, "Localized persistent pulmonary interstitial emphysema in a preterm infant in the absence of mechanical ventilation," Pediatric Radiology, vol. 35, no. 12, pp. 1243-1245, 2005.

[6] P. Gessler, M. Toenz, M. Gugger, and J. Pfenninger, "Lobar pulmonary interstitial emphysema in a premature infant on continuous positive airway pressure using nasal prongs," European Journal of Pediatrics, vol. 160, no. 4, pp. 263-264, 2001.

[7] B. Gurakan, A. Tarcan, I. S. Arda, and M. Coşkun, "Persistent pulmonary interstitial emphysema in an unventilated neonate," Pediatric Pulmonology, vol. 34, no. 5, pp. 409-411, 2002.

[8] M. T. Macklin and C. C. Macklin, "Malignant interstitial emphysema of the lungs and mediastinum as an important occult complication in many respiratory diseases and other conditions: an interpretation of the clinical literature in the light of laboratory experiment," Medicine, vol. 23, no. 4, pp. 281-358, 1944.
[9] A. Y. Bas, N. Okumus, N. Demirel, and A. Zenciroglu, "Persistent pulmonary interstitial emphysema in a preterm infant," Indian Pediatrics, vol. 45, no. 9, pp. 775-777, 2008.

[10] T. E. Wiswell and P. Srinivasan, "Continuous positive airway pressure," in Assisted Ventilation of the Neonate, J. P. Goldsmith and E. H. Karotkin, Eds., pp. 127-147, WB Saunders, Philadelphia, Pa, USA, 4th edition, 2003.

[11] S. Bhojani, D. Bird, and G. Alok, "Spontaneous Diffuse Pulmonary Interstitial Emphysema (PIE) in an unventilated infant," The Internet Journal of Pediatrics and Neonatology, vol. 9, no. 2, 2008.

[12] V. Y. H. Yu, P. Y. Wong, B. Bajuk, and W. Szymonowicz, "Pulmonary interstitial emphysema in infants less than $1000 \mathrm{~g}$ at birth," Australian Paediatric Journal, vol. 22, no. 3, pp. 189$192,1986$.

[13] R. P. Verma, S. Chandra, R. Niwas, and E. Komaroff, "Risk factors and clinical outcomes of pulmonary interstitial emphysema in extremely low birth weight infants," Journal of Perinatology, vol. 26, no. 3, pp. 197-200, 2006.

[14] J. M. Wilson and E. J. Mark, "Case records of the Massachusetts General Hospital. Weekly clinicopathological exercises. Case 30-1997-a preterm newborn female triplet with diffuse cystic changes in the left lung," The New England Journal of Medicine, vol. 337, no. 13, pp. 916-924, 1997.

[15] D. Freysdottir, O. Olutoye, C. Langston, C. J. Fernandes, and N. Tatevian, "Spontaneous pulmonary interstitial emphysema in a term unventilated infant," Pediatric Pulmonology, vol. 41, no. 4, pp. 374-378, 2006.

[16] H. S. Lee and S. A. Im, "Pulmonary interstitial emphysema complicating pneumonia in an unventilated term infant," Indian Journal of Pediatrics, vol. 77, no. 9, pp. 1025-1027, 2010.

[17] S. K. Pursnani, J. B. Amodio, H. Guo, M. A. Greco, and E. P. Nadler, "Localized persistent interstitial pulmonary emphysema presenting as a spontaneous tension pneumothorax in a full term infant," Pediatric Surgery International, vol. 22, no. 7, pp. 613-616, 2006.

[18] D. O’Donovan, M. Wearden, and J. Adams, "Unilateral pulmonary interstitial emphysema following pneumonia in a preterm infant successfully treated with prolonged selective bronchial intubation," American Journal of Perinatology, vol. 16, no. 7, pp. 327-331, 1999.

[19] G. F. Boisset, "Subpleural emphysema complicating staphylococcal and other pneumonias," The Journal of Pediatrics, vol. 81, no. 2, pp. 259-266, 1972.

[20] M. S. Gaylord, R. E. Thieme, D. L. Woodall, and B. J. Quissell, "Predicting mortality in low-birth-weight infants with pulmonary interstitial emphysema," Pediatrics, vol. 76, no. 2, pp. 219-224, 1985. 


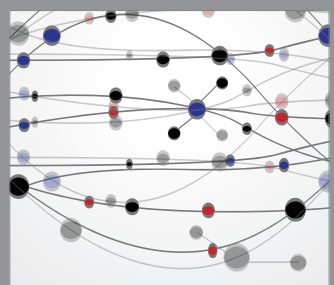

The Scientific World Journal
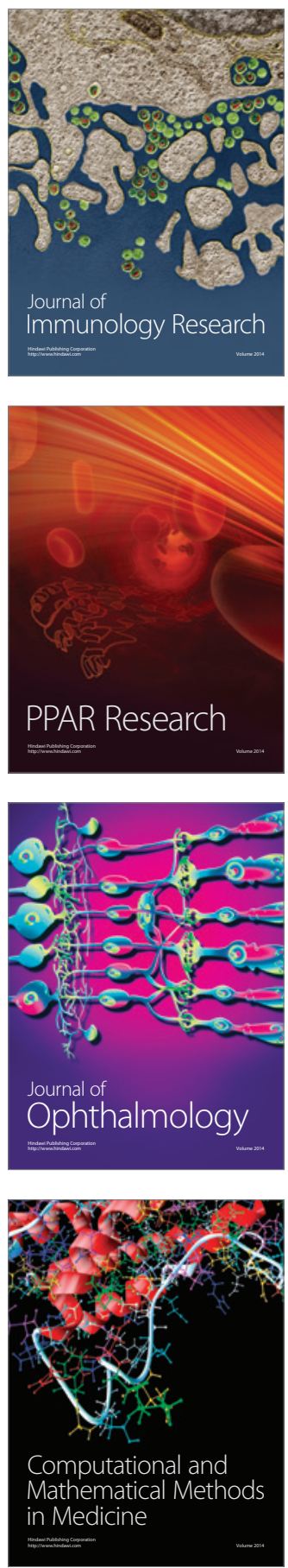

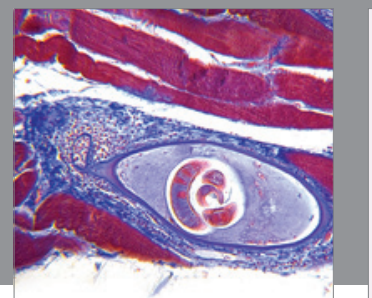

Gastroenterology

Research and Practice
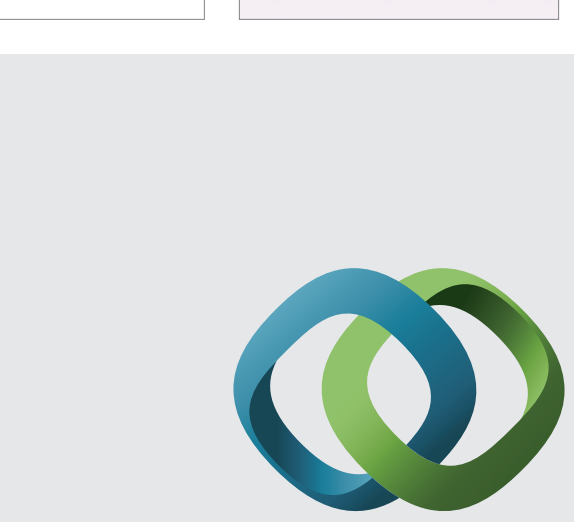

\section{Hindawi}

Submit your manuscripts at

http://www.hindawi.com
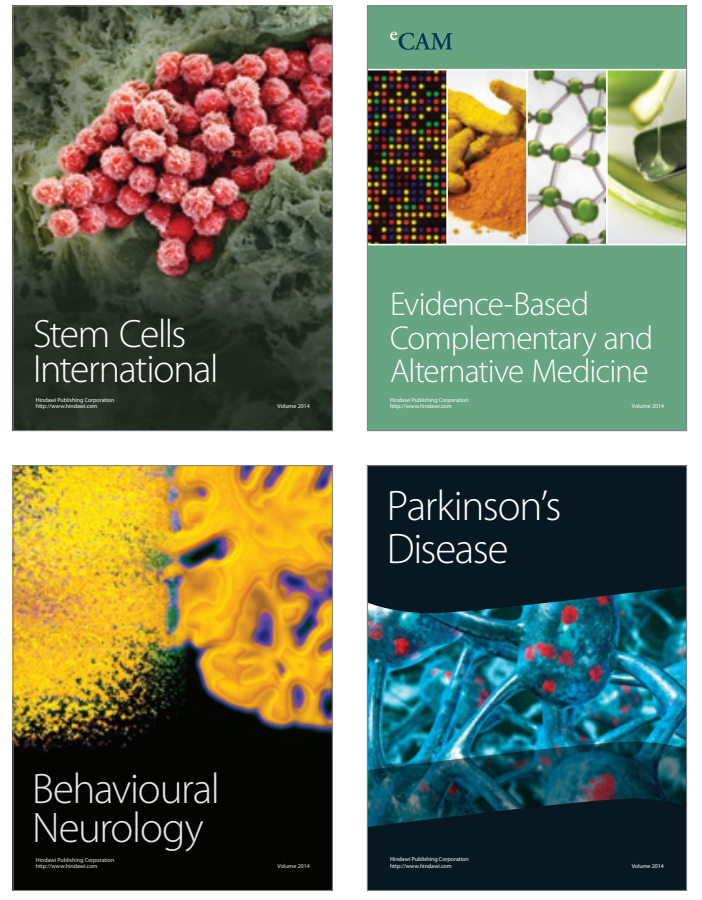
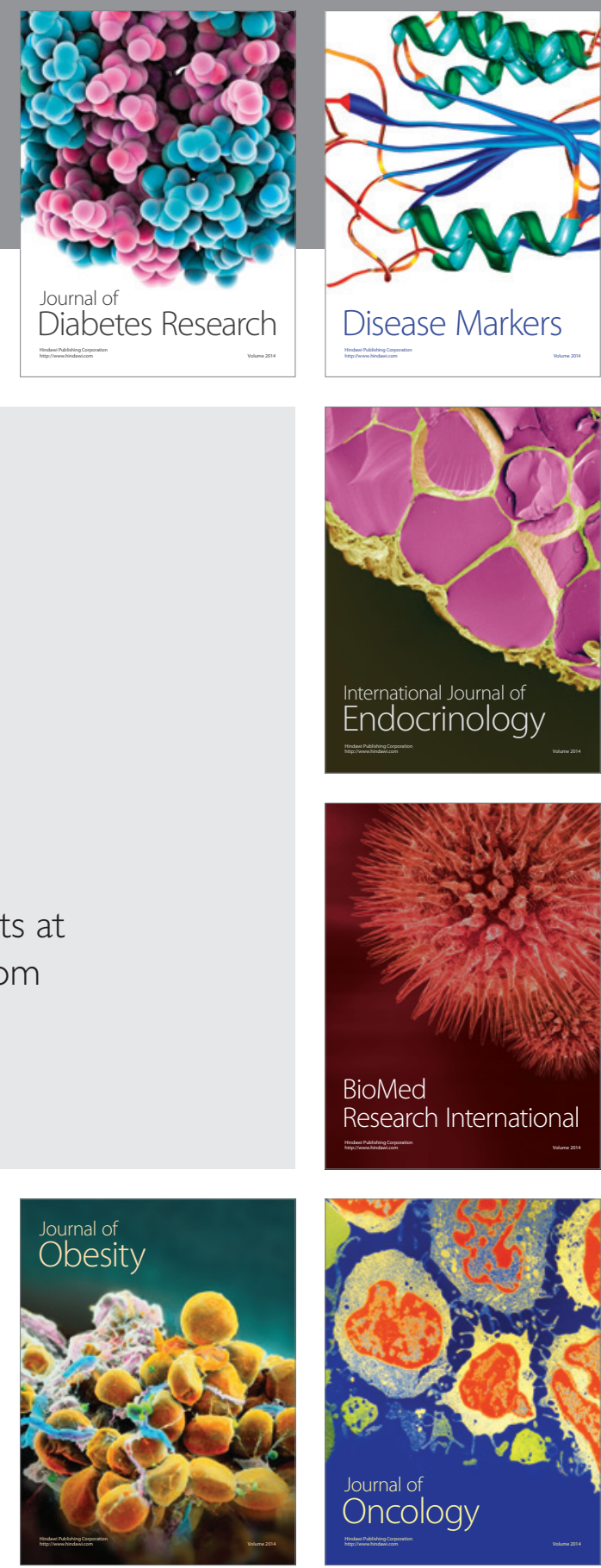

Disease Markers
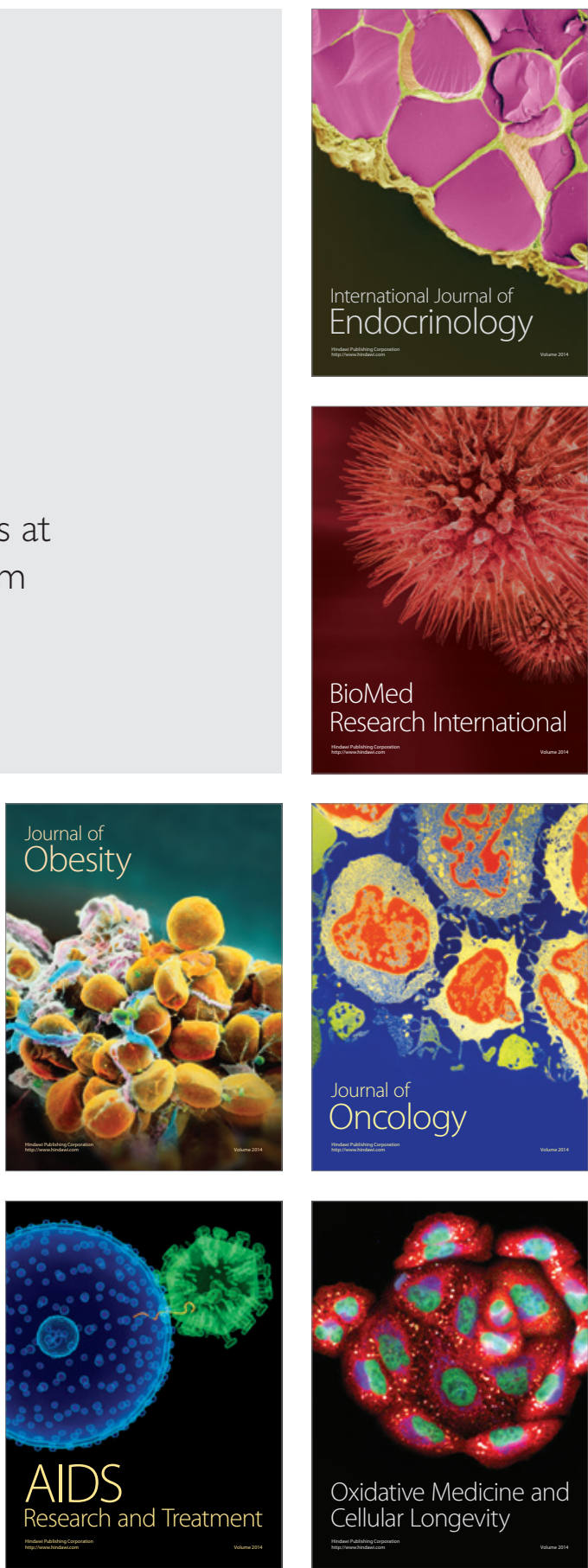